\title{
Bottomia physics at RHIC and LHC
}

\author{
Georg Wolschin ${ }^{1, a}$ \\ ${ }^{1}$ Institut für Theoretische Physik der Universität Heidelberg, Philosophenweg 16, D-69120 Heidelberg, Ger- \\ many, EU
}

\begin{abstract}
In UU collisions at RHIC energies and $\mathrm{PbPb}$ collisions at $\mathrm{LHC}$ energies the suppression of $\Upsilon$ mesons in the hot quark-gluon medium (QGP) versus reduced feeddown is investigated. Our model encompasses screening, collisional damping and gluodissociation in the QGP. For $\Upsilon(1 \mathrm{~S})$ it is in agreement with both STAR and CMS data provided the relativistic Doppler effect and the reduced feed-down from the $\Upsilon(n \mathrm{~S})$ and $\chi_{\mathrm{b}}(n \mathrm{P})$ states are properly considered. At both energies, most of the suppression for the $\Upsilon(1 \mathrm{~S})$ state is found to be due to reduced feed-down, whereas most of the $\Upsilon(2 S)$ suppression is caused by the hot-medium effects. The role of the in-medium effects relative to reduced feed-down increases with energy. The $\Upsilon(1 \mathrm{~S})$-suppression in $\mathrm{PbPb}$ at $\sqrt{s_{\mathrm{NN}}}=5.02 \mathrm{TeV}$ is predicted.
\end{abstract}

\section{Introduction}

The production of heavy mesons and, in particular, of bottomia in initial hard partonic interactions in relativistic heavy-ion collisions at RHIC and LHC energies is of special interest because quarkonia in the fireball can act as a probe to test the properties of the hot medium. The heavier the hadron that is produced in the collision, the shorter its formation time $\tau_{\mathrm{F}}$. Very heavy mesons such as the $\mathrm{J} / \psi$ or the $\Upsilon$ meson in their $1 \mathrm{~S}$ spin-triplet ground states are produced in hard collisions at very short times, typically at $\tau_{\mathrm{F}} \simeq 0.3-0.5 \mathrm{fm} / c$. Since the $\Upsilon(1 \mathrm{~S})$ state is particularly stable, it has a sizeable probability to survive in the hot quark-gluon medium that is produced in the fireball of a heavy-ion collision at LHC energies, even at initial medium temperatures of the order of $400 \mathrm{MeV}$ or above.

There exists meanwhile a considerable literature on the dissociation of quarkonia and in particular of the $\Upsilon$ meson in the hot quark-gluon medium; see [1] and references therein for a review. In [2, 3] we have devised a model that accounts for the gluon-induced dissociation of the various bottomium states in the hot medium (gluodissociation), the damping of the quark-antiquark binding due to the presence of the medium which generates an imaginary part of the temperature-dependent potential, and the screening of the real part of the potential. The latter turns out to be less important for the

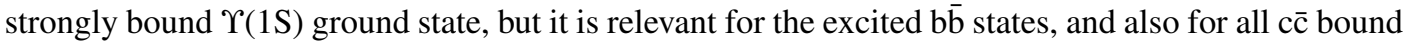
states.

In this work we utilize our model to quantitatively disentangle the role of bottomia suppression in the quark-gluon plasma (QGP) relative to the role of reduced feed-down for the $\Upsilon(1 \mathrm{~S})$ ground state as function of energy, comparing with data from both RHIC and LHC. The relativistic Doppler effect is

a e-mail: wolschin@uni-hd.de 
discussed in detail. We simultaneously consider the $\Upsilon(2 S)$ state where the QGP effects are expected to be much more important with respect to reduced feed-down regarding the measured suppression, and verify this expectation in a calculation. We compare with centrality-dependent CMS data $[4,5]$ for the $\Upsilon(1 \mathrm{~S})$ and $\Upsilon(2 \mathrm{~S})$ states. For $\mathrm{PbPb}$ we predict the centrality-dependent suppression at the higher LHC energy of $\sqrt{s_{\mathrm{NN}}}=5.02 \mathrm{TeV}$.

\section{Relativistic Doppler effect}

The radial Schrödinger equation is solved for the six bottomia states considered in the hot fireball $\Upsilon(1 \mathrm{~S}), \Upsilon(2 \mathrm{~S}), \Upsilon(3 \mathrm{~S})$, and $\chi_{\mathrm{b}}(1 \mathrm{P}), \chi_{\mathrm{b}}(2 \mathrm{P}), \chi_{\mathrm{b}}(3 \mathrm{P})$ - with energies $E_{n l}(T)$ in a complex potential $V_{n l}(r, T)$ and corresponding damping widths $\Gamma_{n l}^{\text {damp }}(T)$ as has been outlined in [3]. We consider the running of the strong coupling in the calculation of the wave function, and the various dissociation processes. Since the coupling constant depends on the solution of the Schrödinger equation for the six bottomium states, we use an iterative method for the solution of the problem [3], together with the one-loop expression for the running of the coupling. In addition to the damping width $\Gamma_{n l}^{\text {damp }}(T)$ we consider the width caused by gluon-induced dissociation $\Gamma_{n l}^{\text {diss }}(T)$ [3]. The total decay width of a given bottomium state is then the incoherent sum $\Gamma_{n l}^{\text {tot }}=\Gamma_{n l}^{\text {damp }}+\Gamma_{n l}^{\text {diss }}$.

Bottomia are too massive to experience a substantial change of their momenta by collisions with the light partons in the medium. Hence, there will be a finite relative velocity between the expanding QGP medium and the $b \bar{b}$ mesons. In the rest frame of the bottomia, the surrounding distribution of massless gluons then appears as a Bose-Einstein distribution with an anisotropic temperature $T_{\mathrm{D}}$ that is determined from the relativistic Doppler effect as

$$
T_{\mathrm{D}}(T,|\boldsymbol{u}|, \theta)=T \frac{\sqrt{1-|\boldsymbol{u}|^{2}}}{1-|\boldsymbol{u}| \cos \theta} .
$$

Here, $\boldsymbol{u}$ is the average velocity of the surrounding fluid cell (measured in the bottomium restframe) and $\theta$ the angle between $\boldsymbol{u}$ and the direction in which the temperature is evaluated.

In the rest frame of the bottomia the Doppler effect causes a blueshifted temperature for $\theta=0^{\circ}$ and a redshifted temperature in the opposite direction $\theta=180^{\circ}$. The effects of red- and blueshift get more and more pronounced with increasing relative velocity $|\boldsymbol{u}|$, but the angular range with $T_{\mathrm{D}}<T$ (redshifted region) is growing while the angular range with $T_{\mathrm{D}}>T$ (blueshifted region) is restricted to smaller and smaller angles $\theta$ as has been noted in [6]. To account for the effect of the anisotropic temperature $T_{\mathrm{D}}$ on the bottomium dissociation we substitute the total decay width $\Gamma_{n l}^{\mathrm{tot}}(T)$ by an effective value

$$
\Gamma_{\mathrm{eff}, n l}^{\mathrm{tot}}:=\left\langle\Gamma_{n l}^{\mathrm{tot}}\left(T_{\mathrm{D}}\right)\right\rangle_{\Omega}=\frac{1}{4 \pi} \int \mathrm{d} \Omega \Gamma_{n l}^{\mathrm{tot}}\left(T_{\mathrm{D}}\right) .
$$

This takes into account the red- or blueshifted temperatures and also correctly describes the nonexistence of bound states once the effective temperature in the blueshifted region exceeds the dissociation temperature, see [7] for more details. The $p_{\mathrm{T}}$-dependence is rather flat and at $2.76 \mathrm{TeV}$ in reasonable agreement with the available CMS data [7]. There is no rapidity dependence in our model, both minimum bias and centrality dependent yields are flat as functions of $y$.

\section{Hydrodynamic expansion}

Our treatment of the hydrodynamic flow of the fireball has been outlined in [3] including transverse expansion. The initial time for the evolution is $\tau_{\text {init }}=0.1 \mathrm{fm} / c$, the initial conditions in the transverse plane $\left(x^{1}, x^{2}\right)$ are given in Eqs. (14) - (16) of [3]. 
We determine the initial central temperature $T_{0}$ from a fit of the experimental $R_{A A}(\Upsilon(1 \mathrm{~S}))$ in central collisions, cf. section 5. It is in reasonable agreement with values derived from ideal hydrodynamical calculations of elliptic flow at RHIC and LHC energies, respectively [8].

The same formation time of $\tau_{\mathrm{F}}=0.4 \mathrm{fm} / c$ is used for ground and exited states, with theta functions for the decay widths of the six states considered. In the co-moving coordinate system used for the hydrodynamical calculation, time dilation of the formation times is taken into account. We had previously investigated the dependence of $R_{A A}$ on $\tau_{\mathrm{F}}$, cf. Fig. 9 in [3].

To estimate the initial populations $N_{A A, n l}^{\mathrm{i}}$ of the six states, we consider the measured final populations $N_{\mathrm{pp}, n l}^{\mathrm{f}}$ of the three $\Upsilon(n \mathrm{~S})$-states in pp collisions at the same energy and calculate the decay cascade [9] backwards to obtain the initial populations in pp, $N_{\mathrm{pp}, n l}^{\mathrm{i}}$ [7] These are then scaled by the number of binary collisions $N_{\text {coll }}$ yielding the initial populations in the heavy-ion case. When the suppression factors are calculated, the number of binary collisions cancels out. The required branching ratios are taken from the Review of Particle Physics [10] or from theory where no experimental values are available (as is the case for $\chi_{\mathrm{b}}(3 \mathrm{P})$ ), see [9] for details and references.

The QGP is approximated as a relativistic perfect fluid consisting of gluons and up-, down- and strange-quarks, described in detail in [3]. We define the QGP-suppression factors $R_{A A, n l}^{\mathrm{QGP}}\left(c, p_{\mathrm{T}}\right)$ which quantify the amount of in-medium suppression of bottomia with transverse momentum $p_{\mathrm{T}}$ for $\mathrm{PbPb}$ collisions in the centrality bin $c$, where $b_{c} \leq b<b_{c+1}$. The QGP-suppression factor is not directly measurable since it accounts only for the amount of suppression inside the fireball due to the three processes of color screening, collisional damping and gluodissociation. It is given by the ratio of the number of bottomia that have survived the fireball to the number of bottomia produced in the collision. The latter scales with the number of binary collisions at a given point in the transverse plane and hence with the nuclear overlap, $N_{\mathrm{b} \bar{b}} \propto N_{\text {coll }} \propto T_{A A}$. We calculate $R_{A A, n l}^{\mathrm{QGP}}$ as outlined in [3].

\section{In-medium suppression versus feed-down}

Once the bottomia states have survived the hot quark-gluon plasma environment, the feed-down cascade from the excited states to the ground state is considered in detail. Due to the rapid melting or depopulation of the excited states caused by the mechanisms in the QGP-phase, the feed-down to the ground state is reduced, resulting in additional $\Upsilon(1 S)$-suppression with respect to the situation in pp collisions at the same energy.

The focus of the present investigation is the determination of the relevance of feed-down for a given bottomium state as function of incident energy (RHIC vs. LHC), and of its relative importance for the $\Upsilon(1 S)$ and $\Upsilon(2 S)$ states, which appears to be a new consideration. As will be shown in the next section, at both RHIC and LHC energies, most of the suppression for the $\Upsilon(1 \mathrm{~S})$ state is found to be due to reduced feed-down, and even more so at the lower RHIC energy. In contrast, most of the $\Upsilon(2 S)$ suppression is caused by the hot-medium effects.

\section{Results and comparison to data}

The suppression of the spin-triplet ground state at both RHIC and LHC energies is well described by our model for initial central temperatures of $T_{0}=417 \mathrm{MeV}$ in UU at $\sqrt{s_{\mathrm{NN}}}=193 \mathrm{GeV}$ and $T_{0}=$ $480 \mathrm{MeV}$ in $\mathrm{PbPb}$ at $2.76 \mathrm{TeV}$, and an $\Upsilon$ and $\chi_{\mathrm{b}}$ formation time of $\tau_{\mathrm{F}}=0.4 \mathrm{fm} / \mathrm{c}$; see Fig. 1 for PbPb, and [7] for UU. The parameters for the density distributions of the lead and uranium ions are taken from [11]. Our minimum bias value of the suppression in $2.76 \mathrm{TeV} \mathrm{PbPb}$ is $R_{A A}^{\min \text {. bias }}(\Upsilon(1 \mathrm{~S}))=0.44$.

The relative contributions of in-medium effects and reduced feed-down change as a function of incident energy, see Tab. 1 for detailed minimum-bias results: In $193 \mathrm{GeV} \mathrm{UU}$, only about $20 \%$ of the 


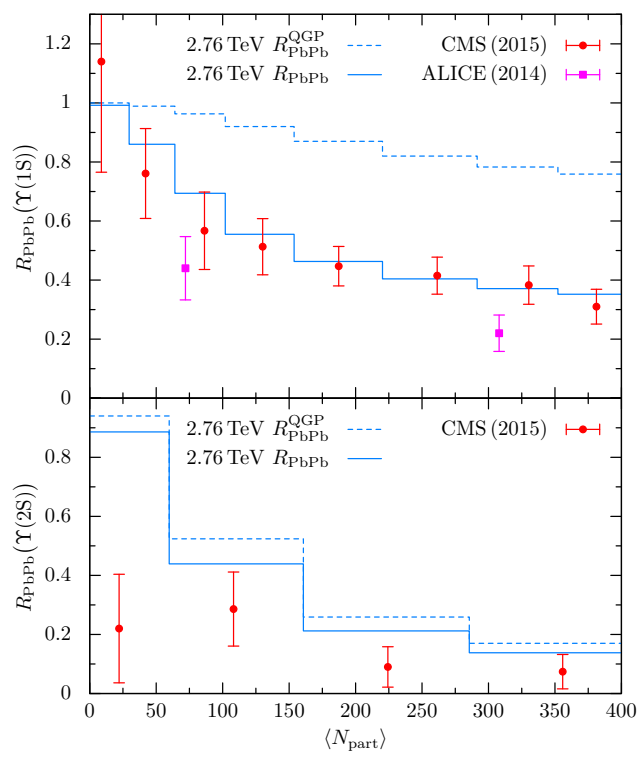

Figure 1. (color online) Top: Calculated suppression factor $R_{A A}(\Upsilon(1 \mathrm{~S}))$ in $\mathrm{PbPb}$ collisions at $\sqrt{s_{\mathrm{NN}}}=2.76 \mathrm{TeV}$ (solid line) together with centrality-dependent data from CMS $(|y|<2.4$, [5]) and ALICE (squares, $2.5<y<$ 4 , [17]) as function of the number of participants $N_{\text {part }}$ (averaged over centrality bins). The suppression factor $R_{A A}^{\text {QGP }}$ in the QGP-phase without the effect of reduced feed-down is shown as dashed (upper) curve. Bottom: Suppression factor for the first excited state $R_{A A}(\Upsilon(2 \mathrm{~S}))$ in $\mathrm{PbPb}$ collisions at $\sqrt{s_{\mathrm{NN}}}=2.76 \mathrm{TeV}$ (solid line) together with preliminary data from CMS [5]. The suppression factor $R_{A A}^{\mathrm{QGP}}$ in the QGP-phase without the effect of reduced feed-down is shown as dashed (upper) curve.

total suppression $\left(1-R_{A A}\right)$ is due to the in-medium effects, whereas in $2.76 \mathrm{TeV} \mathrm{PbPb}$ the in-medium contribution is already about $30 \%$ and further increases in $5.02 \mathrm{TeV} \mathrm{PbPb}$.

The situation is very different for the first excited state $\Upsilon(2 \mathrm{~S})$ as shown in Fig. 1 (bottom) for $2.76 \mathrm{TeV} \mathrm{PbPb}$ : With the same set of parameters as for the $\Upsilon(1 \mathrm{~S})$ state, the calculated suppression of the $\Upsilon(2 S)$ state is much more pronounced in the QGP-phase than for the $\Upsilon(1 S)$ state. Tab. 1 shows that more than $80 \%$ of the total minimum-bias $\Upsilon(2 \mathrm{~S})$ suppression in UU and more than $90 \%$ in $\mathrm{PbPb}$ is due to in-medium effects. Hence the additional contribution of the feed-down cascade on the $\Upsilon(2 S)$ suppression is rather marginal and drops below $10 \%$ at LHC energies. Unfortunately, there are no data available that directly quantify the feed-down fractions as functions of centrality. As is obvious from Fig. 1, the comparison with the CMS data [5] leaves room for additional suppression mechanisms in particular in the peripheral region for the $\Upsilon(2 S)$ state.

For $\Upsilon(1 \mathrm{~S})$ we also predict in Fig. 2 the suppression at the current LHC energy of $5.02 \mathrm{TeV} \mathrm{PbPb}$ as $R_{A A}^{\text {min. bias }}(\Upsilon(1 \mathrm{~S}))=0.40$ using the same formation time, but a scaled initial temperature $T_{0}=513 \mathrm{MeV}$. This value is obtained from the proportionality between the initial entropy density, the charged particle multiplicity per unit of rapidity and the cube of the temperature [12-14]. Here the extrapolated $\mathrm{d} N_{\mathrm{ch}} / \mathrm{d} \eta$ for $0-5 \%$ centrality $\mathrm{PbPb}$ taken from [15] is in agreement with recent data from ALICE [16]. The ensuing enhancement of the suppression is within the experimental error bars of the $2.76 \mathrm{TeV}$ result. It remains to be seen whether this is confirmed by the forthcoming analysis of the mid-rapidity CMS data from LHC Run-II. 
Table 1. Calculated nuclear suppression factors for the $\Upsilon(1 \mathrm{~S})$ and $\Upsilon(2 \mathrm{~S})$ states in minimum-bias $193 \mathrm{GeV} \mathrm{UU}$ as well as 2.76 and $5.02 \mathrm{TeV} \mathrm{PbPb}$ collisions. The in-medium suppression factor is $R_{A A}^{\mathrm{QGP}}$, the total suppression factor including reduced feed-down is $R_{A A}$. The last column gives the percentage of the suppression in the medium relative to the total suppression $\left(1-R_{A A}^{\mathrm{QGP}}\right) /\left(1-R_{A A}\right)$.

\begin{tabular}{rrrcrr}
\hline \hline$\sqrt{s_{\mathrm{NN}}}$ & System & State & $R_{A A}^{\mathrm{QGP}}$ & $R_{A A}$ & $\frac{1-R_{A A}^{\mathrm{QGP}}}{1-R_{A A}}$ \\
\hline $193 \mathrm{GeV}$ & $\mathrm{UU}$ & $\Upsilon(1 \mathrm{~S})$ & 0.919 & 0.568 & $18.8 \%$ \\
$193 \mathrm{GeV}$ & $\mathrm{UU}$ & $\Upsilon(2 \mathrm{~S})$ & 0.469 & 0.399 & $88.4 \%$ \\
\hline $2.76 \mathrm{TeV}$ & $\mathrm{PbPb}$ & $\Upsilon(1 \mathrm{~S})$ & 0.834 & 0.443 & $29.8 \%$ \\
$2.76 \mathrm{TeV}$ & $\mathrm{PbPb}$ & $\Upsilon(2 \mathrm{~S})$ & 0.285 & 0.239 & $94.0 \%$ \\
\hline $5.02 \mathrm{TeV}$ & $\mathrm{PbPb}$ & $\Upsilon(1 \mathrm{~S})$ & 0.783 & 0.396 & $35.9 \%$ \\
$5.02 \mathrm{TeV}$ & $\mathrm{PbPb}$ & $\Upsilon(2 \mathrm{~S})$ & 0.226 & 0.189 & $95.4 \%$ \\
\hline \hline
\end{tabular}

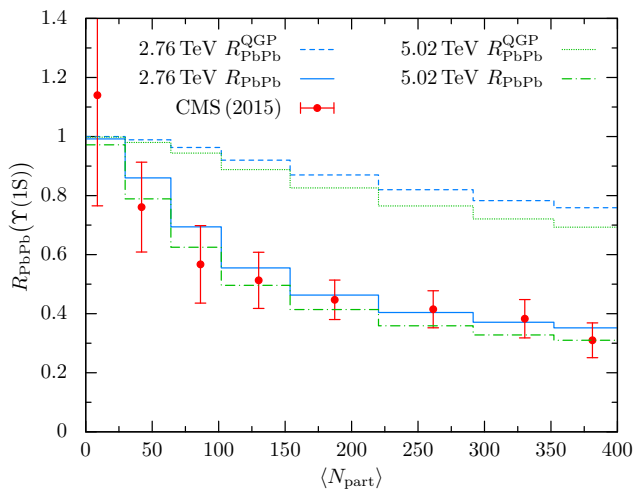

Figure 2. (color online) Calculated suppression factor $R_{A A}\left(\Upsilon(1 \mathrm{~S})\right.$ ) in $\mathrm{PbPb}$ collisions at $\sqrt{s_{\mathrm{NN}}}=2.76 \mathrm{TeV}$ (lower solid line) and prediction at $5.02 \mathrm{TeV}$ (lower dash-dotted line) together with centrality-dependent $2.76 \mathrm{TeV}$ data from CMS $\left(|y|<2.4\right.$, [5]) as function of the number of participants $N_{\text {part }}$ (averaged over centrality bins). The suppression factors $R_{A A}^{\mathrm{QGP}}$ in the QGP-phase without the effect of reduced feed-down are shown as upper curves (dashed and dotted) for both energies, again yielding slightly more suppression at the higher energy.

\section{Conclusion}

In summary, we have investigated the suppression of the $\Upsilon(n \mathrm{~S})$ states in $\mathrm{UU}$ and $\mathrm{PbPb}$ collisions at RHIC and LHC energies in a model that considers the in-medium processes gluodissociation, screening and damping. The feed-down cascade from the excited bottomia states produces substantial additional ground-state suppression, since the excited states melt through screening, or depopulate through dissociation processes and hence, there is less feed-down to the $\Upsilon(1 \mathrm{~S})$ ground state as compared to pp at the same energy. In contrast, the suppression of the first excited state $\Upsilon(2 S)$ at both RHIC and LHC energies is largely due to the properties of the hot quark-gluon medium.

Our model results for the ground state are in agreement with the centrality-dependent STAR and CMS data $[4,5,18]$. The flat transverse-momentum dependence of the suppression factor is consistent with the preliminary CMS data when the relativistic Doppler effect due to the velocity of the moving bottomia relative to the expanding QGP is properly considered. In minimum-bias 
$193 \mathrm{GeV}$ UU, only about $20 \%$ of the total $\Upsilon(1 \mathrm{~S})$ suppression $\left(1-R_{A A}\right)$ is due to the in-medium effects, whereas in $2.76 \mathrm{TeV} \mathrm{PbPb}$ the in-medium contribution is already about $30 \%$ and further increases in $5.02 \mathrm{TeV} \mathrm{PbPb}$.

The suppression of the first excited $\Upsilon(2 \mathrm{~S})$ state which occurs mostly in the QGP-phase requires additional centrality-dependent dissociation mechanisms in particular for peripheral collisions. For the centrality and transverse momentum dependence of $\Upsilon(1 \mathrm{~S})$ we have made predictions at the LHC energy of $5.02 \mathrm{TeV} \mathrm{PbPb}$ where results are currently being analyzed. The ALICE Collaboration has meanwhile released preliminary data for the $\Upsilon(1 \mathrm{~S})$ centrality dependent yields in $5.02 \mathrm{TeV} \mathrm{PbPb} \mathrm{[19]}$ at rapidities $2.5<|y|<4.0$. Here the suppression is found to be slightly less than at $2.76 \mathrm{TeV}$, but almost compatible within the experimental error bars. CMS has presented preliminary $5.02 \mathrm{TeV}$ data in the midrapidity region $|y|<2.4$, but so far only for the double ratio $R_{A A}(\Upsilon(2 \mathrm{~S})) / R_{A A}(\Upsilon(1 \mathrm{~S}))$ [20]. Once their suppressions factors for the individual states are available, the consistency of the ALICE and CMS results, and the agreement with our prediction can be checked.

\section{Acknowledgements}

This work has been partially supported by DFG through TRR 33 at the Universities of Bonn, LMU Munich and Heidelberg. I am grateful to my students - in particular, Johannes Hoelck and Felix Nendzig - for their contributions. An extended version of this conference article is found in [7].

\section{References}

[1] A. Andronic, F. Arleo, R. Arnaldi, A. Beraudo et al., Eur. Phys. J. C 76, 107 (2016)

[2] F. Nendzig, G. Wolschin, Phys. Rev. C 87, 024911 (2013)

[3] F. Nendzig, G. Wolschin, J. Phys. G: Nuclear and Particle Physics 41, 095003 (2014)

[4] S. Chatrchyan et al. (CMS Collaboration), Phys. Rev. Lett. 109, 222301 (2012)

[5] M. Jo et al. (CMS Collaboration), Proc. Quark Matter Conf. Kobe 2015, in preparation; CMSPAS-HIN-15-001 (2015)

[6] M.A. Escobedo, F. Giannuzzi, M. Mannarelli, J. Soto, Phys. Rev. D 87, 114005 (2013)

[7] J. Hoelck, F. Nendzig, G. Wolschin (2016), 1602.00019

[8] B. Schenke, S. Jeon, C. Gale, Phys. Lett. B 702, 59 (2011)

[9] F. Vaccaro, F. Nendzig, G. Wolschin, Europhys. Lett. 102, 42001 (2013)

[10] K.A. Olive et al. (Particle Data Group), Chin. Phys. C38, 090001 (2014)

[11] H. de Vries, C.W. de Jager, C. de Vries, At. Data Nucl. Data Tables 36 p. 495 (1987)

[12] J.D. Bjorken, Phys. Rev. D 27, 140 (1983)

[13] G. Baym, B.L. Friman, J.P. Blaizot, M. Soyeur, W. Czyż, Nucl. Phys. A 407, 541 (1983)

[14] M. Gyulassy, T. Matsui, Phys. Rev. D 29, 419 (1984)

[15] G. Wolschin, Phys. Rev. C 91, 014905 (2015)

[16] J. Adam et al. (ALICE Collaboration), Phys. Rev. Lett. 116, 222302 (2016)

[17] B. Abelev et al. (ALICE Collaboration), Phys. Lett. B 738, 361 (2014)

[18] R. Vértesi et al. (STAR Collaboration), Nucl. Part. Phys. Proc. 276, 269 (2016)

[19] C. Hadjidakis (ALICE Collaboration), Talk at ICNFP2016 Kolymbari, to be published in EPJWeb of Conf. (2016)

[20] Y. Kim et al. (CMS Collaboration), Proc. Hard Probes, Wuhan (2016) 